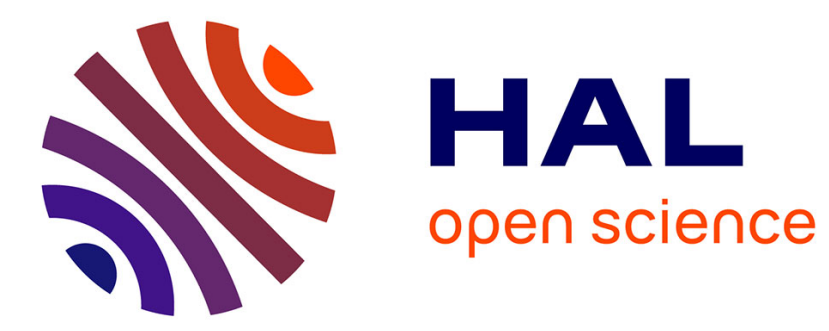

\title{
Shiba Bound States across the Mobility Edge in Doped InAs Nanowires
}

\author{
Alexandre Assouline, Chéryl Feuillet-Palma, Alexandre Zimmers, Hervé \\ Aubin, Marco Aprili, Jean-Christophe Harmand
}

\section{- To cite this version:}

Alexandre Assouline, Chéryl Feuillet-Palma, Alexandre Zimmers, Hervé Aubin, Marco Aprili, et al.. Shiba Bound States across the Mobility Edge in Doped InAs Nanowires. Physical Review Letters, 2017, 119 (9), pp.097701. 10.1103/PhysRevLett.119.097701 . hal-01611648

\section{HAL Id: hal-01611648 \\ https://hal.sorbonne-universite.fr/hal-01611648}

Submitted on 6 Oct 2017

HAL is a multi-disciplinary open access archive for the deposit and dissemination of scientific research documents, whether they are published or not. The documents may come from teaching and research institutions in France or abroad, or from public or private research centers.
L'archive ouverte pluridisciplinaire HAL, est destinée au dépôt et à la diffusion de documents scientifiques de niveau recherche, publiés ou non, émanant des établissements d'enseignement et de recherche français ou étrangers, des laboratoires publics ou privés. 


\title{
Shiba Bound States across the Mobility Edge in Doped InAs Nanowires
}

\author{
Alexandre Assouline, Cheryl Feuillet-Palma, Alexandre Zimmers, and Hervé Aubin \\ LPEM, ESPCI Paris, PSL Research University; CNRS; Sorbonne Universités, \\ UPMC University of Paris 6, 10 rue Vauquelin, F-75005 Paris, France \\ Marco Aprili \\ Laboratoire de Physique des Solides, CNRS, Université Paris-Sud, \\ University Paris-Saclay, 91405 Orsay Cedex, France \\ Jean-Christophe Harmand \\ Centre de Nanosciences et de Nanotechnologies, CNRS, Université Paris-Sud, \\ Universités Paris-Saclay, C2N-Marcoussis, 91460 Marcoussis, France
}

(Received 16 February 2017; published 31 August 2017)

\begin{abstract}
We present a study of Andreev quantum dots fabricated with small-diameter (30 nm) Si-doped InAs nanowires where the Fermi level can be tuned across a mobility edge separating localized states from delocalized states. The transition to the insulating phase is identified by a drop in the amplitude and width of the excited levels and is found to have remarkable consequences on the spectrum of superconducting subgap resonances. While at deeply localized levels only quasiparticle cotunneling is observed, for slightly delocalized levels Shiba bound states form and a parity-changing quantum phase transition is identified by a crossing of the bound states at zero energy. Finally, in the metallic regime, single Andreev resonances are observed.
\end{abstract}

DOI: 10.1103/PhysRevLett.119.097701

In superconductor-quantum-dot-superconductor structures or at impurities in bulk superconductors, Bogoliubovtype bound states can form at an energy smaller than the superconducting gap energy $\Delta$, leading to subgap resonances (SGRs).

The SGR formation depends on the different energy scales: $\Delta$, the coupling $\Gamma_{S}$ of the quantum dot (QDot) with the superconducting electrodes, the charging energy $U$, and the energy $\varepsilon_{0}$ of the QDot level relative to the Fermi energy of the superconducting electrodes. Its phase diagram has been extensively studied theoretically [1,2]. For large coupling $\Gamma_{S}$, the SGRs result from the coherent superposition of multiple Andreev reflections [3,4], and conductance peaks are expected at voltage values $\Delta / n e$ [5], where $n$ is the number of Andreev reflections. For weak coupling $\Gamma_{S}$, where the system is in the regime of Coulomb blockade, the ground state, singlet $|S\rangle$ or doublet $|D\rangle$, results from the competition between the Kondo screening and the superconducting pairing interaction. These two ground states are separated by a parity-changing quantum phase transition, which can be identified by the crossing of the SGRs at zero energy. Previous works have addressed this transition through measurements of Josephson supercurrents [6-10] and studies of the SGRs in S-QDot-S [11-17] or N-QDot-S geometries [18-21]. Recently, similar devices attracted intense interest with the observation of the zero-energy Majorana end states in proximitized nanowires [22-24].

The physics of an odd parity QDot is related to the physics of Shiba states [25-29] forming at magnetic impurities in bulk superconductors, where tuning the magnetic exchange also leads to a parity-changing quantum phase transition [30-36], characterized by a crossing of the Shiba states at zero energy [37].

So far, the impurity-induced superconducting SGRs have been observed only by scanning tunneling microscopy, at Co atoms deposited on $\mathrm{Nb}$ [38], at magnetic impurities in $2 \mathrm{H}-\mathrm{NbSe}_{2}$ [39], and magnetic MnPc molecules deposited on $\mathrm{Pb}$ [37]. However, tunneling spectroscopy of impurity states can also be done with microfabricated devices, such as nanosized Schottky diodes [40], nanosized field effect transistors [41-44], or nanosized memristors [45]. In this work, we present an observation of Shiba bound states forming in a dopant-induced impurity band. In this diffusive regime, it is expected that an Anderson-like metalinsulator transition (MIT) [46] separates the metallic regime at a high carrier concentration from an insulating regime at a low carrier concentration, where the localized and delocalized states in the band structure are separated in energy by a mobility edge. We identified this mobility edge in the conductance spectrum measured as a function of the gate voltage and show that the SGRs are sensitive to the metal-insulator transition through the effect of localization on the coupling parameter $\Gamma_{S}$.

Epitaxially grown InAs nanowires of diameter approximately $d=30 \mathrm{~nm}$ [47] were deposited on a degenerately doped Si substrate covered with a 300-nm-thick thermal oxide. The nanowires are contacted with $e$-beam deposited $\mathrm{Ti}(5 \mathrm{~nm}) / \mathrm{Al}(100 \mathrm{~nm})$ electrodes with moderate in situ ion 
(a)

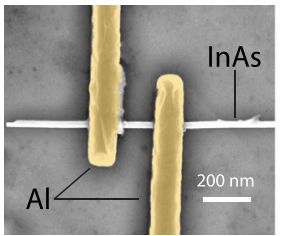

(b)
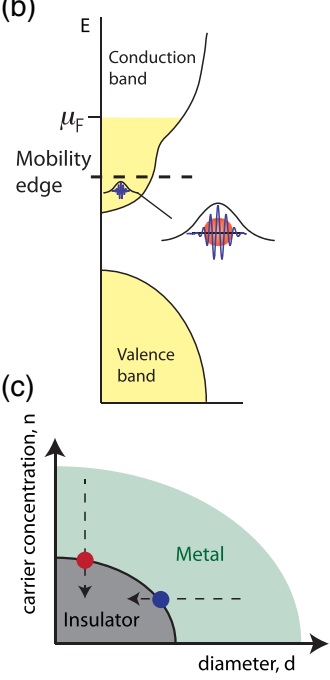
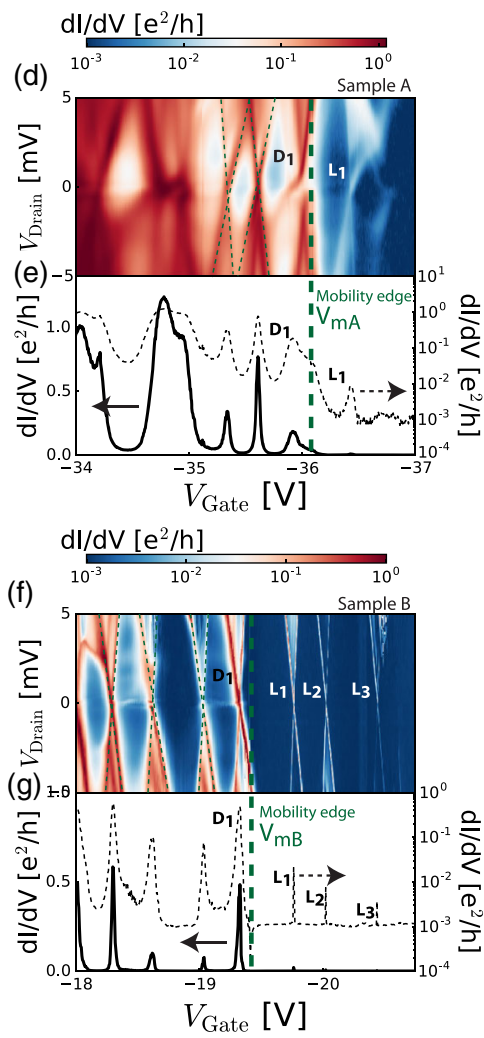

FIG. 1. (a) SEM image of a nanowire device. (b) Sketch of the band structure showing the formation of an impurity band merging with the conduction band. (c) Phase diagram displaying the MIT as a function of the carrier density (red dot) and nanowire diameter $d$ (blue dot). (d), (f) Conductance $d I / d V$ maps for nanowires $A$ and $B$, respectively, as a function of drain bias $V_{\text {drain }}$ and back-gate voltage $V_{\text {gate }}$. Coulomb diamonds are highlighted with dashed lines. These maps show the crossing of the mobility edge with the back-gate voltage, below which only a few excited levels remain visible, labeled $L_{1}$ to $L_{3}$. The last delocalized level for each nanowire is labeled $D_{1}$. (e), (g) Zero-bias conductance as a function of back-gate voltage plotted on the linear scale (continuous line) and the log scale (dashed line).

beam cleaning of the nanowire surface before deposition. Figure 1(a) shows an SEM image of one device. About 20 devices have been fabricated and measured with a standard lock-in method. The room temperature resistivity is below $10 \mathrm{~m} \Omega \cdot \mathrm{cm}$, which is 20 times smaller than the value measured for undoped nanowires of similar diameter [48]. The two-wire and four-wire resistances are similar, indicating negligible contact resistance. Two devices, sample $A$ and sample $B$, have been extensively measured in a dilution fridge with a base temperature of $30 \mathrm{mK}$. Upon cooling, the resistivity increases only up to $15 \mathrm{~m} \Omega \cdot \mathrm{cm}$, which is $2 \times 10^{4}$ smaller than measured on undoped nanowires, indicating metallic behavior as shown now.

Figures 1(d) and 1(f) show conductance maps $d I / d V$ as a function of the drain and gate bias for sample $A$ and sample $B$, respectively. They are measured with a large ac voltage $(\simeq 50 \mu \mathrm{V})$ on a large drain voltage range, making

the superconductinglike features barely visible. They show Coulomb blockade diamonds from which the lever arms $\alpha_{D, S, G}=C_{D, S, G} / C_{\sum}$ and the corresponding capacitances $C_{D, S, G}$ are obtained [49]. The Coulomb energy extracted from the gate voltage separation between nodes $\Delta V_{G}$ is given by $E_{C}=e \alpha_{G} \Delta V_{G} \simeq 2.6 \mathrm{meV}$ for sample $A$ and $E_{C}=4.4 \mathrm{meV}$ for sample $B$.

Upon depleting the nanowire with a negative back-gate voltage, one can identify a threshold, $V_{m A}=-36 \mathrm{~V}$ and $V_{m B}=-19.4 \mathrm{~V}$, for nanowires $A$ and $B$, Figs. $1(\mathrm{e})$ and $1(\mathrm{~g})$, respectively, below which the excitation lines, labeled $L_{1}$ to $L_{3}$, are much narrower and their amplitude much weaker than the excitation lines above the threshold. For nanowire $B$, the amplitude of the Coulomb peak maxima $A_{L_{1}}=$ $A_{L_{2}}=0.12, A_{L_{3}}=0.01$, normalized to the amplitude of the last level $D_{1}$ above the threshold, decreases with the energy of the levels, indicating that the lower-energy levels are more strongly localized. This striking evolution across the threshold indicates that it corresponds to the mobility edge where the states of energy above the mobility edge are delocalized and the states below are localized due to Anderson localization. For both samples, no other excitation levels are observed at gate voltages lower than the localized levels, indicating that the nanowires are indeed completely depleted at these most negative voltages, as shown on the zero-bias conductance measured on a large gate voltage range shown in Fig. S1 [50]. In past works on phosphorus-doped nanosized metal-Si-metal structures [41], dopant levels have also been identified as sharp conductance lines below the pinch-off voltage where the channel is fully depleted.

From these voltage thresholds, an estimation of the carrier concentration at near-zero gate bias is obtained from the relation $n=C_{G} V_{m A(B)} /\left(e l \pi d^{2} / 4\right)$, where $l$ is the distance between the electrodes. Ones find $n_{A}=1.7 \times 10^{18} \mathrm{~cm}^{-3}$ for sample $A(l=100 \mathrm{~nm})$ and $n_{B}=2.1 \times 10^{18} \mathrm{~cm}^{-3}$ for sample $B(l=200 \mathrm{~nm})$. This carrier concentration is 10 times larger than in undoped nanowires [48]. Assuming that each $\mathrm{Si}$ atom provides one electron, each QDot contains $\sim 135$ dopants per $100 \mathrm{~nm}$ unit length. For this density, the average distance between dopants is $\langle r\rangle \simeq 8 \mathrm{~nm}$, which is smaller than the Bohr radius $a_{0} \simeq 30.2 \mathrm{~nm}$, calculated from the relation $a_{0}=4 \pi \varepsilon_{0} \varepsilon \hbar^{2} / m^{*} e^{2}$ with $\varepsilon=15.15$ and the effective mass $m^{*}=0.0265$. In other words, the carrier concentration is much larger than critical density $N_{c}=$ $5.6 \times 10^{14} \mathrm{~cm}^{-3}$ obtained from the Mott criterion [51] $a_{B} N_{c}^{1 / 3} \simeq 0.25$ for metallicity. At this high doping level, an impurity band is expected to form and merge with the conduction band, as sketched in Fig. 1(b), Ref. [52]. As discussed by Mott [51], the Ioffe-Regel criterion for metallicity $\lambda_{F}^{-1} \ell \simeq 1$ implies that a mobility edge separates the delocalized states $\left(\lambda_{F}<\ell\right)$ at a high energy from the Anderson localized states $\left(\lambda_{F}>\ell\right)$ at a low energy. Thus, a MIT is expected either as a function of disorder or carrier concentration as sketched in the phase diagram in Fig. 1(c). 
In nanowires, the amount of disorder is controlled by the diameter, as shown in Ref. [48], where the mobility of undoped nanowires drops abruptly below a critical diameter about $50 \mathrm{~nm}$, which corresponds to a disorder-driven MIT. In contrast, while our nanowires have a diameter smaller than this critical diameter, they are metallic because of their high carrier concentration. They are driven into the insulating regime upon reducing the carrier concentration with the gate voltage.

An indication that the excitation levels observed above the mobility edge are also part of the impurity band comes from the level spacings, which are expected to show large fluctuations as a consequence of the random dopant distribution. The conductance map in Fig. 2(a) for sample $A$ reveals more than 140 Coulomb diamonds measured at a low gate bias above the mobility edge. The corresponding histogram of level spacings is shown in Fig. 2(b). Because the precision on the determination of the level spacing $d \delta \simeq$ $0.2 \mathrm{meV}$ is smaller than the width $\sim 4.2 \mathrm{meV}$ of the histogram, this broad histogram clearly indicates fluctuating level spacing in the nanowire. In this metallic regime, the level spacing distribution is described by the random matrix theory [53,54], which predicts a bimodal Wigner distribution. This distribution is characterized by two maxima. One is at the Coulomb energy $E_{C}$, the other at

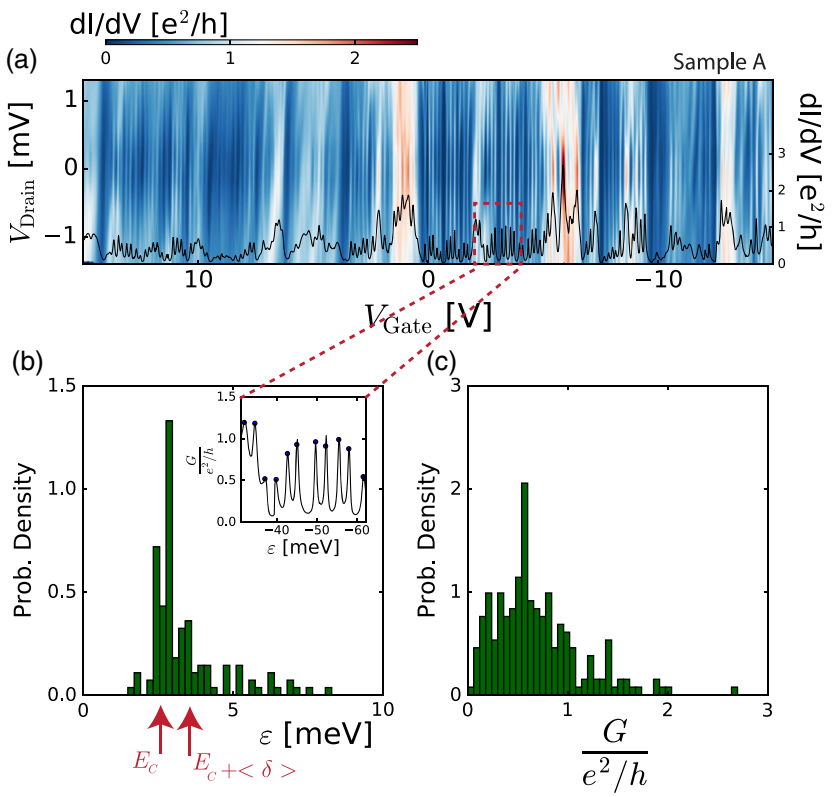

FIG. 2. (a) Conductance $d I / d V$ maps for nanowire $A$ as a function of drain bias $V_{\text {drain }}$ and back-gate voltage $V_{\text {gate }}$. (b) Histogram of level spacing obtained from the energy separation of the Coulomb peaks (inset). As indicated by the red arrows, the main peak of the histogram is located at the charging energy $E_{C}$. A second smaller peak is also expected at $E_{C}+\langle\delta\rangle$. Because of insufficient histogram resolution, a full comparison with the Wigner bimodal distribution has not been attempted. (c) Histogram of the Coulomb peak conductance maxima measured at zero drain bias.
$E_{C}+\langle\delta\rangle$, where $\langle\delta\rangle=1 \mathrm{meV}$ is the mean level spacing calculated from the relation $\langle\delta\rangle=1 / \rho\left(E_{F}\right) V$, where $\rho\left(E_{F}\right)$ is the density of states, $E_{F}=196 \mathrm{meV}$ is the Fermi energy corresponding to the estimated carrier density, and $V$ is the volume of the QDot. The position of these two peaks are indicated in the histogram in Fig. 2(b).

In the random matrix theory, the random distribution of level spacings is accompanied by a random distribution of the amplitude of the wave functions at the boundaries of the QDot, which leads to a broad distribution of the conductance of the Coulomb peak maxima, as observed experimentally and shown in Fig. 2(c). This last observation implies strong fluctuations in the coupling $\Gamma_{S}$ of the levels to the superconducting electrodes which has an important consequence on the formation of the superconducting SGRs. Finally, from the magnetic field dependence of the Zeeman splitting, Fig. S3 [50], a factor $|g| \simeq 10$ is extracted, which is consistent with known values of the $g$ factor in InAs [55].

Figures 3(a) and 3(b) show conductance maps measured at low bias. It reveals the superconducting gap of $\mathrm{Al}$ with four lines symmetric about zero bias. Inside the Coulomb diamonds, the horizontal conductance ridges at $e V_{\text {drain }}=$ $2 \Delta$ correspond to the onset of elastic quasiparticle cotunneling where the peaks in the density of states of the two superconducting electrodes are aligned, as sketched in Fig. 3(e). The superconducting gap value $\Delta \simeq 140 \mu \mathrm{V}$ is extracted, which is similar to values obtained in past works [56]. This figure also shows conductance ridges at $e V_{\text {drain }}=\Delta$. They correspond to $n=1$ Andreev reflections as depicted in Fig. 3(h). They are generally expected in SNS structures $[2,4]$, where the coupling to the electrodes is large, which corresponds to the singlet " 0 " regime in the phase diagram in Fig. 3(h). They have been observed previously in InAs nanowires [12] and carbon nanotubes $[11,13,57,58]$. For certain gate voltages, the pair of Andreev conductance peaks crosses at zero bias, as shown in Figs. 3(c) and S2 [50]. This zero-bias crossing is the consequence of a quantum phase transition with parity change of the S-QDot-S ground state [2] from the singlet 0 state to the doublet $\pi$ state. This transition is expected at low coupling $\Gamma_{S}$ as indicated in the phase diagram in Fig. 3(h). The origin of these occasional zero-bias crossings is the consequence of fluctuating coupling to the electrodes as discussed above. Only those levels which are weakly coupled will show a zero-bias crossing. All these superconductinglike features disappear by the application of a small magnetic field about $20 \mathrm{mT}$.

We now turn to the evolution of the SGRs across the mobility edge. As a consequence of the decreasing coupling $\Gamma_{S}$ of the levels with the superconducting electrodes, the high-resolution enlargement, Fig. 4, shows a remarkable evolution of the SGRs spectrum from the last delocalized level $D_{1}$ to the localized levels $L_{1}$ to $L_{3}$. The level $D_{1}$ shows mostly conductance ridges at $\Delta$. This is 

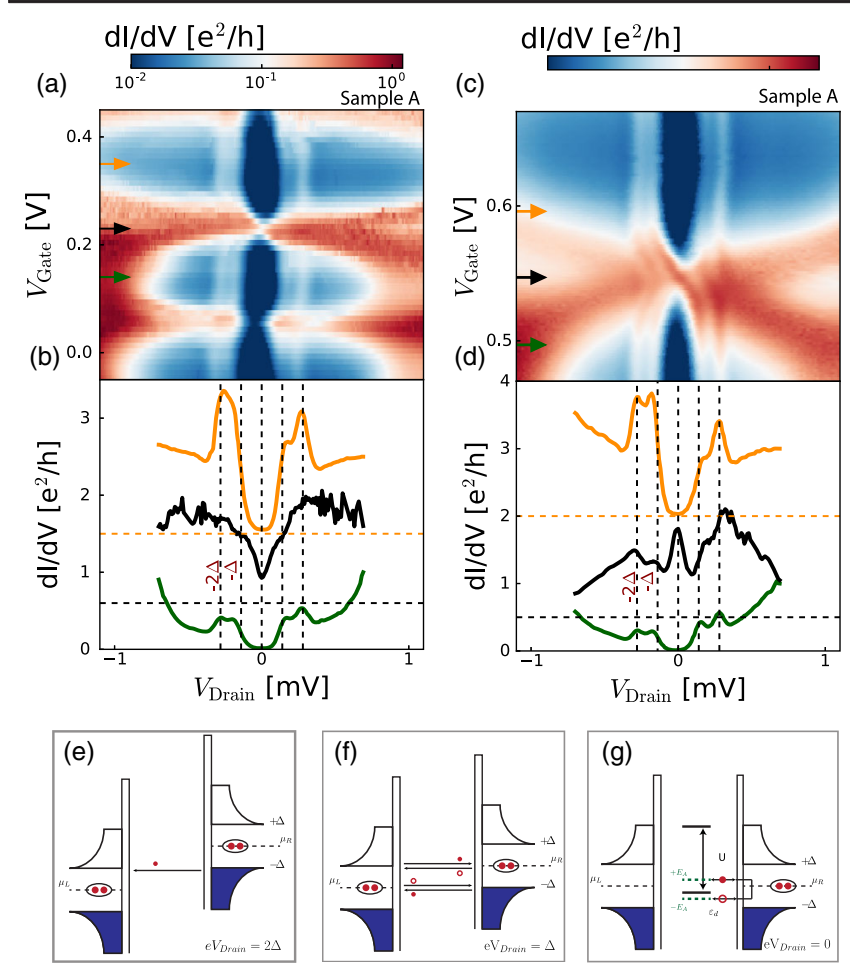

(h)

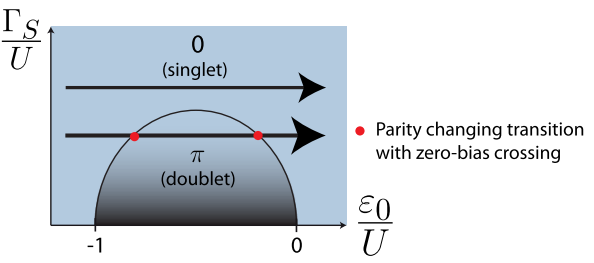

FIG. 3. The conductance maps (a), (c) and the corresponding plot at selected gate voltages (b), (d) show the spectra of the Andreev QDot for two regimes of weak and strong coupling $\Gamma_{S}$, respectively. In the strong coupling regime, only the conductance ridges at $2 \Delta / e$ and $\Delta / e$ are seen. They are, respectively, due to the peak in the density of states at the gap edge (e) and to the firstorder multiple Andreev reflection $(n=1)$ (f). In the weak coupling regime, SGRs form at energies below the superconducting gap energy $(\mathrm{g})$. The parity-changing phase transition is identified by a zero bias crossing of the SGRs. (h) Phase diagram of the S-QD-S system as a function of the charging energy $U$, the QD level $\varepsilon_{0}$, and the coupling to the lead $\Gamma_{S}$. At weak coupling $\Gamma_{S}$, only the singlet ground state can be observed upon changing the gate voltage. At strong coupling, a parity-changing phase transition from the singlet phase to the doublet phase is identified as a zero crossing of the SGRs.

the singlet 0 regime dominated by the $n=1$ Andreev reflections that occur at strong coupling. The first and second localized levels, $L_{1}$ and $L_{2}$, show a crossing of the SGRs at zero energy. This crossing indicates a parity change and the formation of a Shiba resonance at the Si impurity carrying a single electron spin; this is the doublet $\pi$ regime that occurs at weak coupling in the phase diagram in Fig. 3(g). Finally, the last level $L_{3}$ shows only the superconducting gap features at $2 \Delta$, which are the signatures of quasiparticle cotunneling.
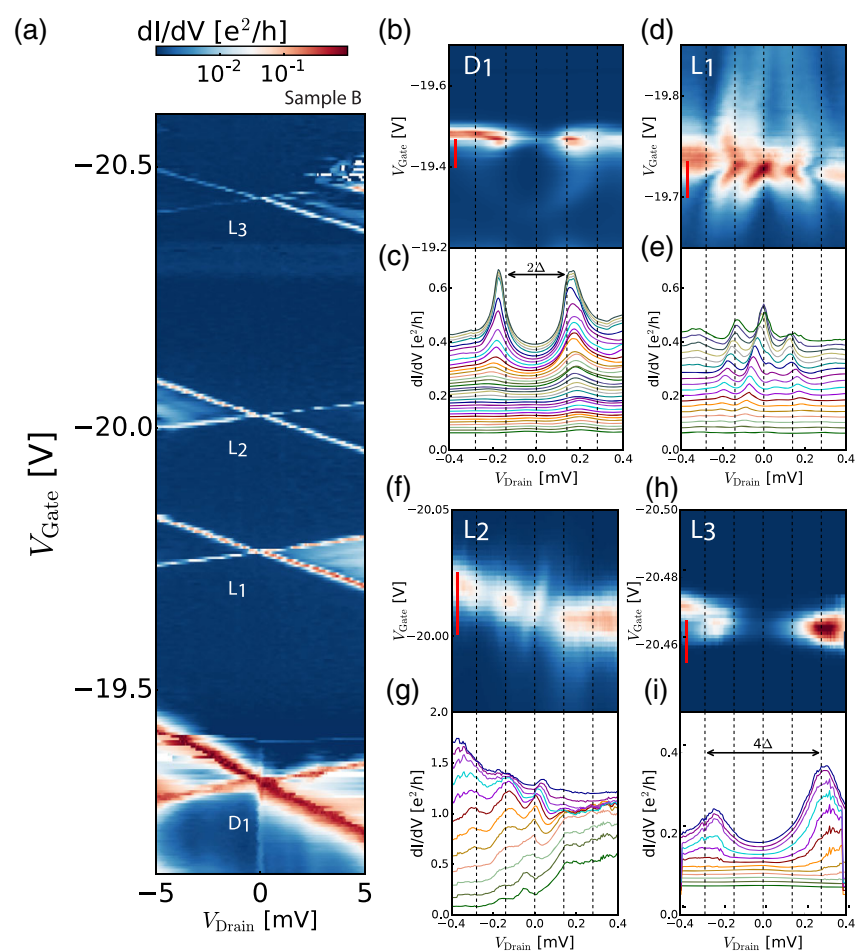

FIG. 4. Conductance map (a) and corresponding enlargements (b), (d), (f), (h) showing the last delocalized level $\left(D_{1}\right)$ and the localized impurity levels $\left(L_{1}, L_{2}, L_{3}\right)$. The waterfall plots are shown for the range indicated by vertical red bars on the corresponding color maps. The shifts between curves are, respectively, of $0.01,0.02,0.1,0.01 e^{2} / h$. On the level $D_{1}$ (b), (c), only conductance peaks at $\Delta$ are observed, which are signature of Andreev reflections $(n=1)$. On the first $L_{1}(\mathrm{~d})$, (e) and second $L_{2}$ (f), (g) localized levels, a crossing of the conductance peak at zero bias is observed, which is the signature of the formation of the Shiba state. Finally, on the last localized level $L_{3}$, only the conductance peaks at $2 \Delta$ are observed, indicating the absence of Andreev reflections and only the presence of quasiparticle cotunneling.

This last level is so localized and weakly coupled to the electrodes that no Andreev reflection occurs in the QDot.

In summary, while past works on InAs-based Andreev QDots employed large diameter nanowires $(>60 \mathrm{~nm})$, we found that Andreev QDots could be fabricated with smaller $(30 \mathrm{~nm})$ diameter nanowires provided that they are initially doped to a high carrier concentration of $2 \times 10^{18} \mathrm{~cm}^{-3}$. We found that these nanowires could be driven across an Anderson MIT upon applying a large negative back-gate voltage, and we observed a remarkable evolution of the superconducting SGRs across the mobility edge as a consequence of the rapidly changing coupling of the levels with the superconducting electrodes. Deeply localized levels do not allow for the presence of Andreev reflections or the formation of Shiba states, and only quasiparticle cotunneling is observed. For localized levels near the mobility edge, Shiba bound states form, and a parity-changing quantum phase transition is identified by a crossing of the SGRs at 
zero energy. Finally, just above the mobility edge, simple $n=1$ Andreev resonances are observed. Further studies of SGRs in disordered QDots could provide insight into the Anderson MIT, which remains a subject of intense theoretical interest $[59,60]$.

We thank A. Descamps-Mandine, M. Rosticher and J. Palomo for their technical support. We acknowledge funding from Labex "Matisse" and Region Ile-de-France in the framework of DIM Nano-K.

*Herve.Aubin@espci.fr

[1] A. V. Rozhkov and D. P. Arovas, Phys. Rev. Lett. 82, 2788 (1999).

[2] A. Martín-Rodero and A. L. Yeyati, Adv. Phys. 60, 899 (2011).

[3] I. O. Kulik, Sov. J. Exp. Theor. Phys. 30, 944 (1969).

[4] M. Octavio, M. Tinkham, G. E. Blonder, and T. M. Klapwijk, Phys. Rev. B 27, 6739 (1983).

[5] E. Scheer, N. Agraït, J. C. Cuevas, A. L. Yeyati, B. Ludoph, A. Martín-Rodero, G. R. Bollinger, J. M. van Ruitenbeek, and C. Urbina, Nature (London) 394, 154 (1998).

[6] J. A. van Dam, Y. V. Nazarov, E. P. A. M. Bakkers, S. De Franceschi, and L. P. Kouwenhoven, Nature (London) 442, 667 (2006).

[7] J.-P. Cleuziou, W. Wernsdorfer, V. Bouchiat, T. Ondarçuhu, and M. Monthioux, Nat. Nanotechnol. 1, 53 (2006).

[8] H. I. Jørgensen, T. Novotný, K. Grove-Rasmussen, K. Flensberg, and P. E. Lindelof, Nano Lett. 7, 2441 (2007).

[9] R. Maurand, T. Meng, E. Bonet, S. Florens, L. Marty, and W. Wernsdorfer, Phys. Rev. X 2, 011009 (2012).

[10] Y. Kanai, R. S. Deacon, A. Oiwa, K. Yoshida, K. Shibata, K. Hirakawa, and S. Tarucha, Phys. Rev. B 82, 054512 (2010).

[11] A. Eichler, M. Weiss, S. Oberholzer, C. Schönenberger, A. Levy Yeyati, J. C. Cuevas, and A. Martín-Rodero, Phys. Rev. Lett. 99, 126602 (2007).

[12] T. Sand-Jespersen, J. Paaske, B. M. Andersen, K. Grove-Rasmussen, H. I. Jørgensen, M. Aagesen, C. B. Sørensen, P. E. Lindelof, K. Flensberg, and J. Nygård, Phys. Rev. Lett. 99, 126603 (2007).

[13] J.-D. Pillet, C. Quay, P. Morfin, C. Bena, A. L. Yeyati, and P. Joyez, Nat. Phys. 6, 965 (2010).

[14] R. S. Deacon, Y. Tanaka, A. Oiwa, R. Sakano, K. Yoshida, K. Shibata, K. Hirakawa, and S. Tarucha, Phys. Rev. Lett. 104, 076805 (2010).

[15] F. Giazotto, P. Spathis, S. Roddaro, S. Biswas, F. Taddei, M. Governale, and L. Sorba, Nat. Phys. 7, 857 (2011).

[16] J.-D. Pillet, P. Joyez, R. Žitko, and M. F. Goffman, Phys. Rev. B 88, 045101 (2013).

[17] A. Kumar, M. Gaim, D. Steininger, A. L. Yeyati, A. Martín-Rodero, A. K. Hüttel, and C. Strunk, Phys. Rev. B 89, 075428 (2014).

[18] T. Dirks, T. L. Hughes, S. Lal, B. Uchoa, Y.-F. Chen, C. Chialvo, P. M. Goldbart, and N. Mason, Nat. Phys. 7, 386 (2011).

[19] R. S. Deacon, Y. Tanaka, A. Oiwa, R. Sakano, K. Yoshida, K. Shibata, K. Hirakawa, and S. Tarucha, Phys. Rev. B 81, 121308 (2010).
[20] E. J. H. Lee, X. Jiang, R. Aguado, G. Katsaros, C. M. Lieber, and S. De Franceschi, Phys. Rev. Lett. 109, 186802 (2012).

[21] W. Chang, V. E. Manucharyan, T. S. Jespersen, J. Nygård, and C. M. Marcus, Phys. Rev. Lett. 110, 217005 (2013).

[22] V. Mourik, K. Zuo, S. M. Frolov, S. R. Plissard, E. P. A. M. Bakkers, and L. P. Kouwenhoven, Science 336, 1003 (2012).

[23] A. Das, Y. Ronen, Y. Most, Y. Oreg, M. Heiblum, and H. Shtrikman, Nat. Phys. 8, 887 (2012).

[24] M. T. Deng, C. L. Yu, G. Y. Huang, M. Larsson, P. Caroff, and H. Q. Xu, Nano Lett. 12, 6414 (2012).

[25] L. Yu, Acta Phys. Chim. Sin. 21, 75 (1965).

[26] H. Shiba, Prog. Theor. Phys. 40, 435 (1968).

[27] A. I. Rusinov, Sov. Phys. JETP 29, 1101 (1969).

[28] T. Soda, T. Matsuura, and Y. Nagaoka, Prog. Theor. Phys. 38, 551 (1967).

[29] H. Shiba and T. Soda, Prog. Theor. Phys. 41, 25 (1969).

[30] A. Sakurai, Prog. Theor. Phys. 44, 1472 (1970).

[31] K. Satori, H. Shiba, O. Sakai, and Y. Shimizu, J. Phys. Soc. Jpn. 61, 3239 (1992).

[32] J. Simonin and R. Allub, Phys. Rev. Lett. 74, 466 (1995).

[33] M. I. Salkola, A. V. Balatsky, and J. R. Schrieffer, Phys. Rev. B 55, 12648 (1997).

[34] A. Balatsky, I. Vekhter, and J.-X. Zhu, Rev. Mod. Phys. 78, 373 (2006).

[35] J. Bauer, A. Oguri, and A. C. Hewson, J. Phys. Condens. Matter 19, 486211 (2007).

[36] W.-F. Tsai, Y.-Y. Zhang, C. Fang, and J. Hu, Phys. Rev. B 80, 064513 (2009).

[37] K. J. Franke, G. Schulze, and J. I. Pascual, Science 332, 940 (2011).

[38] A. Yazdani, C. M. Howald, C. P. Lutz, A. Kapitulnik, and D. M. Eigler, Phys. Rev. Lett. 83, 176 (1999).

[39] G. C. Ménard, S. Guissart, C. Brun, S. Pons, V. S. Stolyarov, F. Debontridder, M. V. Leclerc, E. Janod, L. Cario, D. Roditchev, P. Simon, and T. Cren, Nat. Phys. 11, 1013 (2015).

[40] L. E. Calvet, R. G. Wheeler, and M. A. Reed, Appl. Phys. Lett. 80, 1761 (2002).

[41] H. Sellier, G. P. Lansbergen, J. Caro, S. Rogge, N. Collaert, I. Ferain, M. Jurczak, and S. Biesemans, Phys. Rev. Lett. 97, 206805 (2006).

[42] L. E. Calvet, J. P. Snyder, and W. Wernsdorfer, Phys. Rev. B 83, 205415 (2011).

[43] L. E. Calvet, R. G. Wheeler, and M. A. Reed, Phys. Rev. Lett. 98, 096805 (2007).

[44] L. E. Calvet, R. G. Wheeler, and M. A. Reed, Phys. Rev. B 76, 035319 (2007).

[45] A. Mottaghizadeh, Q. Yu, P. L. Lang, A. Zimmers, and H. Aubin, Phys. Rev. Lett. 112, 066803 (2014).

[46] P. A. Lee, Rev. Mod. Phys. 57, 287 (1985).

[47] F. Glas, J.-C. Harmand, and G. Patriarche, Phys. Rev. Lett. 99, 146101 (2007).

[48] M. Scheffler, S. Nadj-Perge, L. P. Kouwenhoven, M. T. Borgström, and E. P. A. M. Bakkers, J. Appl. Phys. 106, 124303 (2009).

[49] T. Ihn, Electronic Quantum Transport in Mesoscopic Semiconductor Structures, Springer Tracts in Modern Physics (Springer, New York, 2004).

[50] See Supplemental Material at http://link.aps.org/ supplemental/10.1103/PhysRevLett.119.097701 for additional experimnental data including the $g$-factor measurement. 
[51] N. Mott, Metal-Insulator Transitions (Taylor \& Francis, London, 1990).

[52] E. F. Schubert, Doping in III-V Semiconductors (Cambridge University Press, Cambridge, 2015).

[53] Y. Alhassid, Rev. Mod. Phys. 72, 895 (2000).

[54] C. W. J. Beenakker, Rev. Mod. Phys. 69, 731 (1997).

[55] S. Csonka, L. Hofstetter, F. Freitag, S. Oberholzer, C. Schönenberger, T. S. Jespersen, M. Aagesen, and J. Nygård, Nano Lett. 8, 3932 (2008).

[56] C. Buizert, A. Oiwa, K. Shibata, K. Hirakawa, and S. Tarucha, Phys. Rev. Lett. 99, 136806 (2007).
[57] M. R. Buitelaar, W. Belzig, T. Nussbaumer, B. Babić, C. Bruder, and C. Schönenberger, Phys. Rev. Lett. 91, 057005 (2003).

[58] K. Grove-Rasmussen, H. I. Jørgensen, B. M. Andersen, J. Paaske, T. S. Jespersen, J. Nygård, K. Flensberg, and P. E. Lindelof, Phys. Rev. B 79, 134518 (2009).

[59] D. Belitz and T. Kirkpatrick, Rev. Mod. Phys. 66, 261 (1994).

[60] F. Evers and A. Mirlin, Rev. Mod. Phys. 80, 1355 (2008). 